R. J. Cohen and W. T. Sullivan, III, eds.

\title{
Origin of Major L-Band Interference Received by the HALCA Space Radio Telescope
}

\author{
S. Yu. Lioubtchenko and M. V. Popov \\ Astro Space Center FIAN, Profsoyuznaya str. 84/32, Moscow 117810, \\ RUSSIA
}

\author{
H. Hirabayashi and H. Kobayashi
}

\begin{abstract}
Institute of Space and Astronautical Science 3-1-1 Yoshinodai Sagamihara Kanagawa 229-8510 JAPAN
\end{abstract}

\begin{abstract}
About 40 hours of observing data received by the space radio telescope HALCA at L-band $(1.6 \mathrm{GHz})$ were analyzed in order to investigate interference received by the space radio telescope. Autocorrelation spectra for this study were specially prepared at the DRAO S2-correlator with a $7.8125 \mathrm{kHz}$ frequency resolution in each $16 \mathrm{MHz}$ channel. It was found that during $20 \%$ of the observing time the interfering signal was above the tolerable level of $1 \%$ of total receiver noise in a $16 \mathrm{MHz}$ channel. The major source of interference is identified with uplink communication from ships to geostationary satellites in the International Maritime Satellite service (INMARSAT). The frequency range allocated for INMARSAT is $1636.5-1645.0 \mathrm{MHz}$. INMARSAT uses four geostationary satellites, two of which are located above the Atlantic Ocean where the strongest interference was observed. To avoid this interference it is recommended to move the HALCA observing frequency range from the currently used $1634-1666 \mathrm{MHz}$ to $1645-1677 \mathrm{MHz}$. A simple criterion is proposed to predict harmful interference from INMARSAT. This criterion may be used in scheduling of future HALCA observations at L-band.
\end{abstract}

\section{Introduction}

In February 1997 the first space-VLBI radio telescope HALCA was launched into elliptical orbit around the Earth by the Japanese M-V rocket from the Kagoshima Space Center of the Institute of Space and Astronautical Science. Thus, space radio astronomy entered a new era. The satellite's orbit has a revolution period of 6.3 hours, an inclination of $31.4^{\circ}$ and an eccentricity of 0.60 . The $8 \mathrm{~m}$ radio telescope changes its height relative to Earth's surface from 560 to $21400 \mathrm{~km}$. HALCA's very sensitive receiving systems allow radio interferometric observations at $1.60-1.73 \mathrm{GHz}$ (L-band) and $4.7-5.0 \mathrm{GHz}$ (C-band).

From the first observations it became clear that the space radio telescope quite often received significant interfering signals at L-band. However, interference with the same frequency distribution and time behavior was never reported as being observed at ground radio observatories. The purpose of this study was 
to identify the origin of the main interference received by HALCA at L-band and to develop recommendations for the planning of future interferometric observations with space-ground radio interferometers.

\section{Observations}

The autocorrelation spectra for our analysis were prepared by Brent Carlson at the Dominion Radio Astrophysical Observatory (DRAO) Correlator using 2048channel frequency resolution in each main $16 \mathrm{MHz}$ channel $(7.8125 \mathrm{kHz}$ resolution) and an integration time of $500 \mathrm{~s}$. The total observing time was about 40 hours with the spacecraft being at quite different positions in its orbit relative to the Earth's continents. Therefore, we can consider the results of our study statistically meaningful. In our analysis we paid attention only to major interfering signals which might be harmful for interferometric observations. Only harmonics with amplitudes greater than $50 \sigma$ were taken into account (where $\sigma$ is the root-mean square deviation in those $7.8125 \mathrm{kHz}$ channels free from interference).

Figure 1 shows a spectrum damaged by strong interference whose integrated power constitutes $30 \%$ of the total noise power received from the sky in the lower of HALCA's two $16 \mathrm{MHz}$ channels; the calibration tones, at $1 \mathrm{MHz}$ intervals, are just visible.

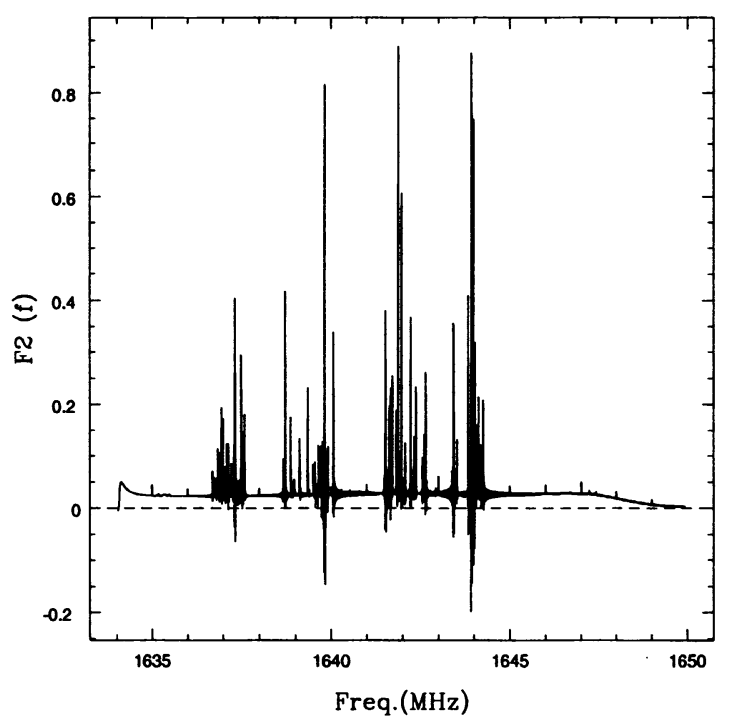

Figure 1. Example of spectrum with strong harmful interference.

In the following sections we will analyze the main parameters of the observed interference in order to identify its source(s). 


\section{Parameters of the Observed Interference}

\subsection{Frequency Distribution}

The frequency distribution of the observed interference is shown in Figure 2. One can see that the major portion is located in the range $1634-1645 \mathrm{MHz}$. In this report we will consider only this interference, since interference observed in other ranges was never found to be harmful for continuum interferometric observations.

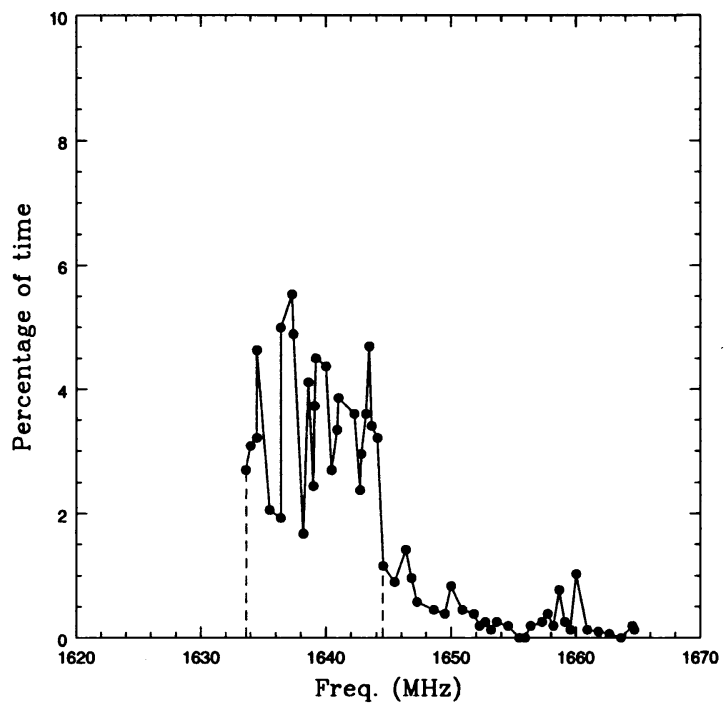

Figure 2. Frequency distribution of the observed interference.

\subsection{Power Distribution}

Figure 3 presents the percentage of time when the interference was greater than a given percentage of the total receiver noise power in a $16 \mathrm{MHz}$ channel. For Very Long Baseline Interferometry (VLBI) observations the tolerable interference level is determined by the requirement that the power level of the interfering signal should not exceed $1 \%$ of the receiver noise power, as set forth in Recommendation ITU-R RA 769. One can see from Figure 3 that in about $80 \%$ of the observing time the interfering signal did not exceed the $1 \%$ limit. To keep the figure compact, the rightmost box (of $5 \%$ height located between 10 and $11 \%$ of interference power) includes in fact interfering signals as strong as $40 \%$. This whole box corresponds to a single occasion when the interfering signal was that high: October 11, 1997, from 19:40 till 21:20 UT (experiment code v101e). For the upper channel $(1650-1666 \mathrm{MHz})$ the interfering signal never exceeded the tolerable level of $1 \%$.

\subsection{Geographical Coverage}

To understand the origin of the interference we calculated the position of the HALCA satellite in its orbit for the experiments under consideration. For this 


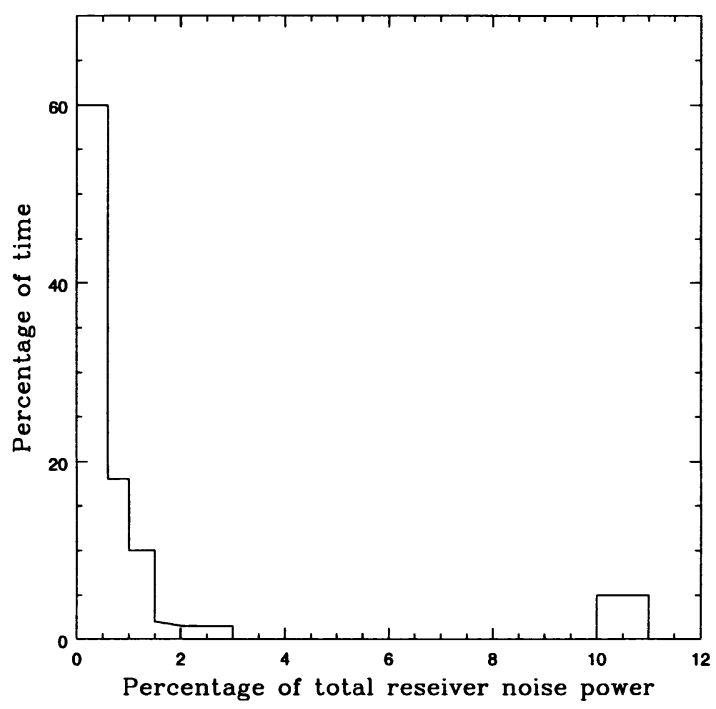

Figure 3. Power distribution of the observed interference.

purpose we used a Keplerian orbit with the osculating elements corresponding to the date of the observation. We found the accuracy of this orbit approximation quite sufficient for our purposes by comparing our calculation of HALCA tracking passes with those used for mission operations. We have not found any correlation between the strength of the interference and the spacecraft position above a particular continent. There was also no correlation between the power of the interfering signal and the height of the satellite above the Earth's surface.

\subsection{Relative Velocities of HALCA and the Interfering Sources}

The frequency resolution of the autocorrelation spectra was $7.8125 \mathrm{kHz}$, which corresponds to a Doppler shift of $1.43 \mathrm{~km} \mathrm{~s}^{-1}$. This resolution was sufficient to distinguish between two possible origins of interference: Earth stations or space stations. It was found by visual inspection of the time-frequency diagrams produced with this frequency resolution that the interfering signals appear as a regular sequence of harmonics with a spacing of $50 \mathrm{kHz}$. Such regular structures in the spectra permit us to investigate the time behavior of the relative velocity between the source of interference and the spacecraft. Because of the large number of harmonics involved, the accuracy of the velocity measurements was as high as $0.1 \mathrm{~km} \mathrm{~s}^{-1}$. We compared the calculated radial velocity of HALCA as seen from the Earth's centre and the velocity behavior of the interfering signal. It was evident that the source of interference was located at the Earth's surface. For a geostationary satellite, and even more so a low orbit satellite, the velocity curves would have been very different!

As a result it was not difficult to find the communication system responsible for the interfering signals received by HALCA at L-band: it is the INMARSAT International Maritime Satellite service which provides communication for the maritime industry. 


\section{General Characteristics of INMARSAT Communication Service}

INMARSAT provides a complete range of ship-to-shore and shore-to-ship communications via geostationary satellites. At present, INMARSAT serves about 50000 Mobile Users (MU) with voice, fax, E-mail and telex services.

INMARSAT's geostationary satellites (Space Stations - SS) are located at the following ranges of longitude: $178^{\circ} \mathrm{E}, 179^{\circ} \mathrm{E}$ (Pacific Ocean Region); $54^{\circ} \mathrm{W}$, $55^{\circ} \mathrm{W}$ (Atlantic Ocean, Region-West); $15.5^{\circ} \mathrm{W}, 17^{\circ} \mathrm{W}$ (Atlantic Ocean, RegionEast); $64.5^{\circ} \mathrm{E}, 65.5^{\circ} \mathrm{E}$ (Indian Ocean Region). There are about 20 Ground Stations (GS) communicating with the SS and connecting calls from ships to the ground network. Uplink communications from ships to satellites (MU to SS) produce interfering signals for HALCA at L-band. There are 339 frequency channels in the range $1636.5-1645 \mathrm{MHz}$; the bandwidth of a single channel is about $12 \mathrm{kHz}$ and the central frequency of any channel is given by

$$
f_{n}=1636.5+0.025 * n \mathrm{MHz} .
$$

There are many kinds of MU stations, providing an EIRP (effective isotropic radiated power) of $37.0 \mathrm{dBW}$ in an $8.5 \mathrm{MHz}$ band. Antenna diameters of $\mathrm{MU}$ stations vary from $0.6 \mathrm{~m}$ to $2.0 \mathrm{~m}$. For example, the $1.3 \mathrm{~m}$ antenna for the Russian MU station "Volna" has the following beam pattern (Zhilin, 1988):

$$
\begin{aligned}
& H B W D=8^{\circ} ; \quad G_{t}=24 \mathrm{~dB} \text { at } \theta=0^{\circ} ; \quad G_{t}=8 \mathrm{~dB} \text { at } 16^{\circ}<\theta<21^{\circ} ; \\
& G_{t}=41-25 * \log \theta \mathrm{dB} \text { at } 21^{\circ}<\theta<57^{\circ} ; \quad G_{t}<3 \mathrm{~dB} \text { at } \theta>57^{\circ} .
\end{aligned}
$$

Using the simple relation for free space transmission loss

$$
d P=P_{t} G_{t} G_{r}(\lambda / 4 \pi D)^{2}
$$

one can estimate the necessary separation distance $D$ between a single MU and HALCA when the interfering signal from this single MU would not be harmful. The HALCA radio telescope at $L$-band has a maximum gain of $39.7 \mathrm{~dB}$, its zero gain is at $23^{\circ}$, and the gain is reduced to $-28 \mathrm{~dB}$ for angles greater than $90^{\circ}$. Let us consider that the angle between the HALCA beam and the direction to the $\mathrm{MU}$ is always greater than $90^{\circ}$, and calculate the separation distance for three different positions of the HALCA spacecraft relative to the MU communicating with the SS:

\begin{tabular}{|c|c|}
\hline $\begin{array}{c}\text { Angle between HALCA } \\
\text { and SS as seen from MU }\end{array}$ & $\begin{array}{c}\text { Distance beyond which } \\
\text { interference tolerable }(\mathrm{km})\end{array}$ \\
\hline$\theta=0^{\circ}$ & 15000 \\
\hline $16^{\circ}<\theta<21^{\circ}$ & 2700 \\
\hline$\theta>45^{\circ}$ & 1000 \\
\hline
\end{tabular}

In these calculations we assumed the tolerable level of interference for continuum VLBI observations in a $16 \mathrm{MHz}$ bandwidth at L-band to be equal to $-156.5 \mathrm{dBW}$, as determined in our previous research (Popov 1996).

It is evident that when HALCA is in the main beam of a MU it would receive harmful interference at nearly any portion of its orbit even from the single MU, while it is safe from harmful interference at angles greater than $30^{\circ}$. Of course, the real situation is even worse when we take into account the density of mobile users and the operational load of the INMARSAT service. 


\section{Conclusion}

The frequency allocations in the HALCA L-band observing range are shown in Figure 4, where "RA" denotes the frequency bands allocated for radio astronomy.

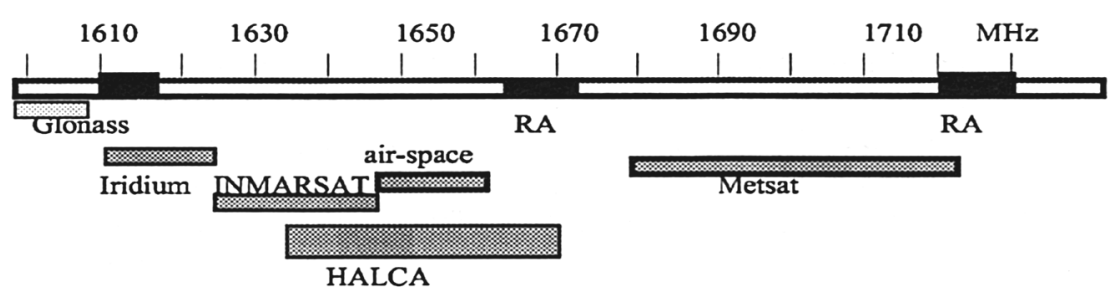

Figure 4. Frequency allocations in the HALCA L-band observing range.

The best frequency allocation for HALCA continuum measurements seems to be $1645-1677 \mathrm{MHz}$. This frequency range overlaps with the air-fleet to space communication band $(1646.5-1660 \mathrm{MHz})$, which was not found to be harmful for HALCA. Overlap with the Metsat band (1675-1710 MHz) may be harmful for ground observatories. In fact, there were reports of interference around $1680 \mathrm{MHz}$ and $1690 \mathrm{MHz}$ at Narrabri (Australia) which was strong enough to double the system temperature in a $64 \mathrm{MHz}$ band. At the VLBA significant interference has been occasionally observed in the $1674-1679 \mathrm{MHz}$ frequency range. Also at the VLA frequencies lower than $1660 \mathrm{MHz}$ suffer less from interference.

If the current frequency allocation for continuum observations with HALCA at L-band $(1634-1666 \mathrm{MHz})$ remains the same, then a prediction of the expected interference from INMARSAT should be included in the scheduling of observations.

We would like to emphasize that space close to the Earth (especially the volume inside the geostationary radius of $36000 \mathrm{~km}$ ) is very dangerous for radio astronomy because of numerous telecommunication systems and services, and careful selection of the bands for radio astronomy must be underaken in developing such space radio astronomy projects.

Acknowledgments. We gratefully acknowledge the VSOP Project, which is led by the Japanese Institute of Space and Astronautical Science in cooperation with many organizations and radio telescopes around the world. We are also grateful to Brent Carlson, who prepared data for our analysis at the DRAO S2 Correlator in Penticton, and to Phil Edwards for his remarks and corrections.

\section{References}

Zhilin V.A. "International Maritime Satellite Service INMARSAT", Handbook, pp.1-159, Sudostroenie, Leningrad, 1988 (in Russian)

Popov M.V. "Estimates of Potential Radio Interference for VSOP from Space Related Systems", Memo ASC FIAN 1439/1, ISAS/VSOP 003/1, 1996 